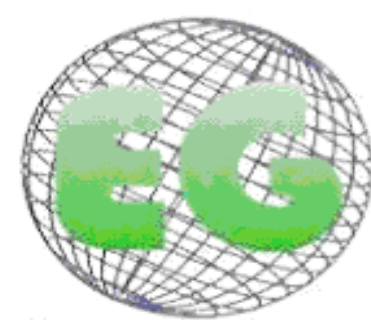

ISSN 1695-6141 $\mathbf{N}^{\circ} 22$
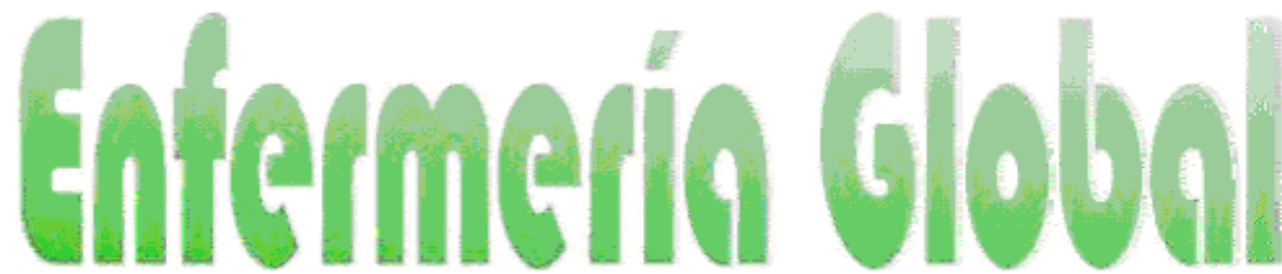

Revista electrónica trimestral de Enfermería

Abril 2011

www.um.es/egloball

\title{
TÓPICOS Y ARQUETIPOS DE LA OPINIÓN PÚBLICA: LA CONSTRUCCIÓN SOCIAL DE LA ENFERMERA
}

\author{
CLICHÉS AND ARCHETYPES IN PUBLIC OPINION: THE SOCIAL \\ CONSTRUCTION OF NURSING
}

*Games, María Inés

*Enfermera. PhStudent. Comunicación y Periodismo. Gabinete de Comunicación y Educación. Universidad Autónoma de Barcelona.

Palabras clave: Mujer, Feminismo, Enfermera, Opinión pública, Arquetipo, Estereotipo.

Keywords: Woman; Feminism; Nurse, opinion publishes; Archetype; Stereotype

\section{RESUMEN}

La indagación realizada intenta mostrar el sufrimiento y menosprecio de las mujeres en un trayecto de la historia.

Concateno el ser mujer y su relación con la disciplina enfermera, por ser la enfermería desde sus ancestros, una actividad netamente femenina. La mujer enfermera unida a la religión, en otras circunstancias, concebidas socialmente como mártires. En determinadas épocas socio culturales, importa a la sociedad transmitir las buenas costumbres sociales de las mujeres. El corolario denominador radica en el sentimiento de las mujeres en brindarse a otros para sentirse útiles y así ayudar a la sociedad.

Se cita a damas que indujeron a cambios como mujeres y que hicieron historia como "mujeres" en la actividad de enfermería.

Se muestra la fuerza de la opinión pública en la construcción social de arquetipos y estereotipos instituidos por la sociedad con respecto a la mujer y a la enfermera.

Se menciona al feminismo como un movimiento social a favor de las mujeres, algunas concepciones y por ser el movimiento provocador en los cambios socioculturales de las mujeres. Mujeres, en general, condenadas a la sumisión y a la vida privada, las que resurgen y participan en la vida pública.

\section{ABSTRACT}

The inquiry attempts to show the suffering and neglect of women in a period of history. It links being a woman and her relationship with the nursing discipline, as nursing from the outset has been an essentially feminine activity. The female nurse, united with religion, in other instances was conceived socially as a martyr. In certain socio cultural times it has been socially important to transmit the good social habits of women. The corollary denominator is the feeling of giving of themselves to others in order to feel useful and to help society. 
It makes mention of women who as "women" introduced changes of historical importance in the nursing profession.

It shows the strength of public opinion in the construction of archetypes and stereotypes in society in terms of the nurse and women.

Feminism is mentioned as a social movement for women, along with some concepts and the fact that it is a movement that provoked socio-cultural changes for women.

Women in general condemned to submission and privacy, but who break through and participate in public life.

\section{Introducción}

El presente trabajo se cimenta en la indagación de la imagen de las mujeres, y como corolario el tópico y arquetipo de la opinión pública sobre la enfermera. Intento evidenciar cómo históricamente la mujer se convierte en un colectivo social en desventaja.

Comprender el género de ser mujer es como la que no existe, porque existen las mujeres como individuos particulares. El primer problema de la cultura es intentar introducir a la mujer en un prototipo. Es entender una a una en el contexto social para no caer en prejuicios, las mujeres se insertan en grupos socioculturales que determinan actitudes y comportamientos en diferentes situaciones vitales durante la vida.

El género mujer, valorado como la condición de menosprecio que se puede utilizar con respecto a lo femenino. La idea de concebir la noción de género femenino, como concepto explicativo para entender la subordinación de la mujer y, por consiguiente, la enfermera, al ser históricamente la profesión eminentemente femenina. Existen un conjunto de argumentos y conceptos que constituyen la perspectiva feminista que sostienen la plataforma para entender la desventajosa situación de la mujer a lo largo de la historia.

Argumentación que perdura desde hace más de tres siglos que describe los fenómenos y que procura explicar las causas que siguen trasladando a la mujer a ocupar una situación de subordinación y desventajas.

Desde la perspectiva feminista ${ }^{1}$ intento crear un marco interpretativo que ponga de manifiesto el género como una estructura de poder, ya que la teoría feminista constituye un paradigma en el que se aplica una mirada intelectual específica, utilizando ciertos conceptos de género, androcentrismo, sistema sexo-género y otros.

El sistema sexo - género ${ }^{2}$ forma parte del instrumento conceptual de la teoría feminista y de los estudios de identidad de género coexistiendo como una construcción social, factor de subordinación y desventaja que, históricamente, la condición anatómica ha sido un argumento sobre la que se ha fundamentado el concepto de identidad femenina. No significa tener el sexo opuesto al hombre, sino que representa someterse a un conjunto de normativas y a ocupar determinados espacios sociales. Este sistema revela el mecanismo que permite la distribución desigual de los recursos culturales, políticos, económicos con acento en el género femenino, siendo el hombre quien tiene un sistema de poder compuesto por los recursos y posición social que le permite ejercer el dominio en diversos terrenos socioculturales. 
La sociedad ha creado un sistema de legitimaciones para justificar la diferencia sexual, por lo tanto la identidad y desigualdad de género son una construcción social de lo masculino y lo femenino, manifestándose de diferentes formas culturales y disímiles sociedades como el común denominador que divide a la sociedad por la mitad. ${ }^{3}$

A las mujeres se las engloba en tareas como en el matrimonio, la maternidad y los cuidados que corresponden a un espacio meramente privado, mientras que los hombres se desarrollan en el ámbito público acentuadamente patriarcal. Incitaciones para el movimiento feminista del siglo XVIII, expresión social de los roles de género en los que se hacen manifiestos las desigualdades entre ambos géneros.

El feminismo ${ }^{4}$ hace visible las estructuras sociales y sus mecanismos ideológicos que colocan a las mujeres en una situación de discriminación constante en los variados ámbitos sociales. Además, pone al descubierto la posición de los varones, el hombre que ocupa un lugar de poder en un sistema social que le permite en forma hegemónica dominar la sociedad en diferentes esferas.

El feminismo entendido como marco interpretativo es también considerado uno de los movimientos sociales que ha logrado una mayor democratización de la sociedad, reivindicando los derechos de las mujeres en la participación civil y política, en los debates públicos donde se insertan cuestiones morales y políticas reprimidas por las instituciones patriarcales. (Ej.: la invisibilidad de la economía doméstica $)^{5}$.

El análisis reflexivo nos permite ver que el feminismo es uno de los efectos sociales que más ha contribuido para la modernidad en el progreso social y político del último siglo centrado a favor de las mujeres.

\section{Breve reseña histórica del contexto de las mujeres}

Históricamente, las mujeres dedicadas a la especificidad de su condición anatómica. En este encierro histórico la humanidad ha perdido con la cultura de la inferioridad y su consiguiente sumisión femenina que atravesó la historia y las conciencias que han afectado hasta el siglo $\mathrm{XX}{ }^{6}$

A las mujeres se las despojó del derecho a la existencia propia, se atacó su dignidad personal y, por supuesto, se les negó el derecho a la educación y acceso a las ciencias, el arte, la música, la literatura, la pintura...

El Derecho Romano lo muestra en la expresión fragilitas sexus que incapacitó a las mujeres, jurídica y cívicamente. Durante siglos quedaron encuadradas como imbecillitas, infirmitas conceptuadas como el sexo débil. ${ }^{7}$

Nietzsche ${ }^{8}$ de la forma más posesiva y desgarrada expresó: "El (hombre) debe considerar a la mujer como propiedad, un bien posible que es necesario poner bajo llave, un ser hecho para la domesticidad y que no tiende su perfección más que a una situación subalterna". Se pensó que era lo justo y necesario, que quedaran encerradas, guardasen la casa, la 
honestidad. Pocas mujeres se quejaron y la mayoría se resignó, interiorizaron su situación y se paralizaron.

Galdós en el siglo XIX aseguraba que "el mayor encanto de la mujer reside en su ignorancia". La evolución de la construcción social la quería sufrida, casta, cuidadosa, cuidadora, benigna, piadosa, obediente, callada y recogida.

Los movimientos sociales (feminismo) provocan una apertura en el camino conforme se comienzan a diluir las barreras existentes entre las mujeres y las autoridades, religiosa, educativa, política, jurídica y económica.

A partir de la segunda mitad del siglo XX se inicia una renovación en las silenciosas actividades de las mujeres, en el ámbito social se comienza a atender su voz, cada vez con mayor protagonismo y dando lugar a nuevas formas de lectura del pasado histórico. Las mujeres fueron excavando y reconstruyéndose en nuevos pilares de resistencia y sufrimiento.

Cada cultura atribuye características, expectativas y comportamientos diferentes para cada sexo, surgiendo los arquetipos como modelos ideales de actuación de los individuos .En la socialización de los individuos surgen los estereotipos como necesidad de preservar ciertas normas sociales dando razón a la existencia.

\section{Tópicos y arquetipos de la construcción social de la enfermera}

El arquetipo ${ }^{9}$ entendido como fuente, patrón, origen, impresión, modelo del cual otros objetos, ideas o conceptos se derivan. Para la filosofía de Platón, el arquetipo expresa la forma sustancial, ejemplares eternos y perfectos de las cosas que existen eternamente en el pensamiento divino.

Los arquetipos habitan en el mundo mítico de los sueños, son modelos o tipos ideales que reúnen las condiciones sociales esenciales y características únicas. Existen arquetipos femeninos y femeninos con abundancia de relaciones e interpretaciones.

Cada época y sociedad formulan nuevas versiones de arquetipos, con rasgos, atributos y tipos de conductas que abarcan comportamientos y actitudes, relacionados al modelo y a la interacción social temporal.

El estereotipo ${ }^{10}$ es la derivación del arquetipo con propiedades simbólicas, reproducción desvirtuada con prefabricado mental y un sinfín de copias que se corresponden con la discriminación y los prejuicios. Contrariamente al arquetipo, el estereotipo puede seguir operando sin contenido sólido y como una reproducción desvirtuada.

\section{La construcción social de la enfermera}

Mujeres a cargo de enfermos, sumamente responsables, protectoras, eficientes en su tarea de sanación, a veces solitarias, las que realizan su trabajo con pasión, con dedicación y algún sufrimiento que connota espiritualidad para resistir su labor. 
Mujeres que atienden enfermos en tiendas de campaña o en hospitales donde las religiosas enfermeras eran las responsables del arte de curar, labor que realizaban como caridad y bajo la dirección de un médico.

La opinión pública afirma que todos necesitan de la enfermera, habitualmente es conocida en su hábitat y popular en su contexto. Su accionar no busca reconocimiento, solo cumplir con su deber, los moribundos piden su presencia y ella se conmueve con el dolor ajeno. Se porta bien con los demás, la relacionan con la maternidad, la denominan religiosa, madre, hermana o madre enfermera como impulsora de algo que imprime fuerza y vitalidad.

La madre enfermera precedió al mago curandero (curalotodo, hechicero o brujo). La enfermera es la persona que atiende enfermos, la que realiza la práctica más antigua que la medicina, es la que asiste a los médicos (hombre, construcción social del arquetipo y estereotipo).

La enfermera es la samaritana que realiza cuidados en la sala de curas, en el hospital, en el manicomio o casa de socorro, cuida y cura con disciplina para la sanación. Concebida como samaritana, de Samaría, por ser compasiva y ayuda a quien la necesita. También es vista por la sociedad como mártir, por su dedicación a la asistencia, por su paciencia, su fe, la esperanza, valentía y el amor a Dios.

Ediht Stein, ${ }^{11}$ (1891-1942) en 1914 (Guerra Mundial) siente la necesidad de ser útil y se alista como enfermera en un hospital de Austria. Carmelita y mártir, años después es nombrada Patrona de Europa y elevada a los altares por el Papa Juan Pablo II en Colonia, el 1 de mayo de 1997.

Agostina Petrantoni ${ }^{12}$, (1864-1894) fue mártir de la caridad y beatificada en 1972 por el Papa Pablo VI .En el año 2003 es nombrada Patrona de las Enfermeras

Otra referencia trascendente en la construcción social de la enfermera, fue Florence Nightingale ${ }^{13}$, nacida en Florencia, pero viviendo gran parte de su vida en Inglaterra (18201910). Considerada la madre de la enfermería, fue quien marcó la historia y el punto de partida en habilidades matemáticas, el magisterio, el espíritu y la disciplina. Mujer pública que perteneció a la época victoriana.

Definió a la enfermedad como el camino que utiliza la naturaleza para desembarazarse de los efectos o condiciones que han interferido en la salud. Puntualizó que la salud no es meramente estar bien, sino ser capaz de usar la energía que poseemos.

Nightingale especificó que la salubridad de las viviendas tienen en cinco puntos a tener en cuenta: aire y agua, desagües eficaces, limpieza y luz, cinco conceptos fundamentales para no quebrar la salud. 
Fue innovadora en la recolección, tabulación, interpretación y presentación gráfica de estadísticas descriptivas. Perteneció a la Sociedad de Estadística Real (1858) y miembro de la Asociación Americana de Estadística (1874). Su principal publicación (1959) tiene el título de "Notas sobre Enfermería", pero además, fue la creadora del primer marco conceptual de enfermería.

Otra mujer intercontinental: la conocida Cecilia Grierson ${ }^{14}$, la primera mujer que se graduó en medicina en América del Sur. Obtuvo el título de médico en 1889, en la Universidad de Buenos Aires. Fundó la primera Escuela de Enfermería en Argentina, en Buenos Aires, bajo su dirección hasta 1913. Definida feminista práctica y equilibrada que luchó en Europa y Argentina por la elevación social e intelectual de la mujer.

\section{Esencia y presencia de la enfermera}

El uniforme y los símbolos son elementos constitutivos de los arquetipos y estereotipos que construyen un mecanismo cognitivo que participa en la construcción del conocimiento y funcionamiento de la memoria, operan como dispositivo dinámico para el aprendizaje con el que construimos la realidad contextual. Forman parte de una estructura mental que permite elaborar construcciones culturales y su significado se emana del contexto relacional en el que está inmerso.

El correcto uso del uniforme da la buena apariencia que inspira confianza y es socialmente respetado porque dignifica y significa el conjunto de cualidades que tienen las enfermeras y que dan a entender que poseen disciplina y una esmerada atención límpida hacia sus semejantes, tanto del alma como del cuerpo.

La vestimenta en color blanco es color femenino por excelencia que se asocia a sentimientos y cualidades. Las religiosas enfermeras vestían de blanco como color de virtudes espirituales hincadas en el amor, la nobleza y la caridad.

El bien, lo limpio, lo esterilizado, el color de la luz y la mortaja de los muertos son de color blanco. Lo que es higiénico, cuidar enfermos, los antiguos mobiliarios hospitalarios también blancos, como sinónimo de limpieza y pureza.

La capa utilizada por las enfermeras, de fieltro para protegerse de las bajas temperaturas en las guardias de 24 horas. Se imponía en las ceremonias para hacerles sentir el peso en sus hombros de la responsabilidad contraída con la sociedad por haber elegido ser enfermera para brindar cuidados, amor y dedicación.

La lámpara de las enfermeras se utilizó para observar el estado de los enfermeros en las rondas nocturnas. F. Nightingale fue la precursora durante la guerra de Crimea (1854), inmortalizada por Longfellow como "La Dama de la Lámpara."

La cofia o toca, de color blanco, se remonta a la Edad Media y su fin fue cubrir el cabello. La cofia considerada como signo de identidad, ideológico, para establecer diferencias y expresarse como pertenencia grupal y territorial. 
El contexto sociocultural creó el Día Mundial de la Enfermera ${ }^{16}$ celebrado el 12 de mayo y en honor a la pionera de la profesión, Florence Nightingale que estaba convencida que el mundo necesita además de inteligencia, bondad y comprensión.

\section{Evolución histórica contextual de la mujer enfermera}

Las mujeres dedicadas a trabajar de enfermeras pasaron años con luchas intestinas, de cambios, con un antes y un después, un antes como ejercicio doloroso, a veces indeseable pero muy humanitario. En esta evolución histórica, cantidad de mujeres sufrieron costes excesivos porque consagraron su vida al cuidado, con un después, cada vez más científico y humanitario.

Inicialmente y por siglos la actividad de ser mujer y enfermera se fundamentaba sutilmente por prédicas maximalistas que solo daban crédito a las normas dolorosas de una ética que exigía permanentes sacrificios y renuncias. Las mujeres dedicadas a ser enfermeras sufrían la sumisión que a partir del siglo XX comienza la emancipación para establecerse como profesión liberal.

La nueva proyectiva de la enfermera surge durante la formación y con el aporte e interacción de las ciencias biológicas, naturales, sociales, psicológicas, se construye un conocimiento propio que la enfermera valida permanentemente en su ejerció profesional con el apoyo, perfeccionamiento de la técnicas y habilidades enmarcadas en el proceso de enfermería.

Nace una práctica de salud más humana, más integral con una proyectiva profesional con estética para el desarrollo y sostenibilidad del fenómeno de la mujer como enfermera porque posee y tiene diversos accesos, se respeta su autonomía, sus creencias personales, conocimientos, experiencias, actualizaciones.

Los arquetipos se presentan en abundancia de relaciones e interpretaciones, con dualidades que pueden sesgarse en lo positivo y en lo negativo del estereotipo con una función sancionadora social a modo de cualidades deseables o indeseables. Lo positivo o lo negativo son construcciones sociales centradas en la fábrica individual de prejuicios y estereotipos resultados de la interacción y socialización. ${ }^{17}$

\section{Conclusiones}

A finales del siglo XIX nace una etapa nueva para las mujeres quienes, paso a paso, pretenden apropiarse de identidad propia, social, cultural, económica y laboral.

No es necesario ser feminista para insertarse como mujer, pero el feminismo, en evolución creciente, alega cada vez más en sus plataformas la defensa de un nuevo concepto de mujer en diferencia por naturaleza con el hombre, con aspecto trascendente pero necesariamente reubicando a la mujer en lo social, en lo laboral, en la política y en la economía.

La mujer interrumpe con los mandatos que determinaron su existencia privada, invisible, excluida y minimizada, comienza a compartir otros escenarios, otros terrenos socio demográficos, con competencia intelectual, política, económica y socio cultural, y se (re)funda en su espacio de acción, como mujer pública y visible con inclusión social. 
La mujer involucra a la enfermera y la enfermera a la mujer, considerando el esquema tradicional del trabajo, el superior o el opuesto es el hombre, el médico (dominante) y la mujer, la enfermera en inferioridad (sumisión), el invento del catálogo social de la época que, por asociación, la mujer cuida y la enfermera cuida a enfermos y heridos.

La opinión pública formalizó los estereotipos a partir de la concepción de la madre enfermera, el padre médico, por lo tanto, el hijo es el paciente.

El médico considera que por su estatus no debía estar al lado del enfermo o del herido, sólo esporádicamente brindarle atención y cierto control de su evolución.

La enfermera, mujer que cuida y asiste, realiza las curas menores, es quien debe permanecer prolongadamente cerca del enfermo o herido.

Durante la segunda guerra mundial aparece la figura de la enfermera científica, en interacción con las enfermeras estadounidense, conceptualizadas como enfermeras tecnológicas, mujeres geográficamente diferenciadas dan origen a las especialidades en la aplicación de tratamientos y en el canon curativo.

Edith Stein y Agostina Pietroni marcaron una etapa para la enfermera y para la mujer. Florence Nightingale, como impulsora, innovadora y líder demostró al hombre que sus teorías, conocimientos y prácticas tenían validez argumental concretándolo desde un posicionamiento no feminista. La construcción histórica social muestra a la pionera británica, admirada por su colectivo enfermero, perdura como objeto de estudio en la esfera académica, no solo por su postura, su cientificismo, que la sobrevive en el imaginario social. Cecilia Grierson en América, Buenos Aires, Argentina, provocó la inserción de la mujer, mediada por la educación en enfermería.

En esta evolución conceptual, contextual y práctica se conceptualizan los cuidados enfermeros basados en diagnósticos de enfermería con sus respectivos y autónomos tratamientos decididos por mujeres.

La dependencia médico-enfermera-enfermera-médico, puede perpetuarse o no, sólo desde la órbita farmacológica por ser el médico quien da la indicación.

El origen de este oficio inicial destinado a la mujer, cambia sustancialmente, se retroalimenta a través del tiempo, nosotras, las protagonistas somos parte del discurso e integrantes de la sociedad. Y la sociedad, en cambio constante, es la que intencionalmente construye, constituye, cambia y define las nuevas estructuras sociales como condición o consecuencia del discurso.

Los espacios públicos tanto del hombre como el de la mujer, en pugna persistente aún en el siglo XXI, en esta evolución se construyen los arquetipos y estereotipos, entre las luchas de poder, aunque exista, entre el hombre y la mujer, la diferencia anatómica por naturaleza.

Mi reflexión final, con las palabras de A. Tofler, las que nos ayudan a comprender los tópicos y arquetipos de la opinión pública con respecto: a la evolución de la mujer y de la disciplina enfermera. 
"Las huellas en la mente son más importantes que las de la luna. Se reconoce ahora, tardíamente, que comienza a emerger sobre el planeta una nueva economía revolucionaria basada en el conocimiento"

\section{Bibliografia / Webliografia}

1. Varela, Nuria. Feminismo para principiantes. Consultar en: http://www.femitec.com

2. Bourdie, Pierre. Manual de Información de Género. Instituto de la mujer. Madrid. 2006

3. Op. Cit. Feminismo

4. Op.cit. Feminismo

5. Op.cit. Feminismo

6. Consultar en: http://www.vallenajerillo.com

7. Graziosi, Marina. Infirmitas sexus. En: http://www.pensamientopenal.com.ar/dossier/

8. Nietzsche, Friedrich. http://www.literato.es/nietzsche frases voluntad/

9. Definición de arquetipo. En: http://es.wikipedia.org/arquetipo

10. Perceval Verde. José María. Seminario de Tópicos y Arquetipos. Facultad de Ciencias de la Comunicación. Doctorado en Ciencias de la Comunicación y Periodismo. Universidad Autónoma de Barcelona. 2006

11.Stein, Edith. En: http://es...wikipedia.org.EdithStein

12. Pietrantoni,Agostina.En: http://pqsanvicentect.org/Santos; En: http://es.wikillingue.com/fr/AgostinaPietrantoni

13. Nightingale, Florence. En: http://es.wikipedia.org/wiki/FlorenceNightingale

En: http://www.ibe.unesco.org/publications/ThinkersPdf/nightins.PDF; En: http://ciencia.astroseti.org/matematicas/articulo 3755 biografia florence nightingale.htm

14. Grierson, Cecilia. Una visionaria del siglo XIX. En:

http://www.mujereshoy.com/secciones/1004.shtml; Grierson, Cecilia. Biografía. En: http://ceciliagrierson.org/biodra.htm 
15. Nightingale, Florence. En: http://www.ibe.unesco.org/publications/ThinkersPdf/nightins.PDF

16. Nightingale, Florence. En: http://ciencia.astroseti.org/matematicas/articulo $\underline{3755 \text { biografia florence nightingale.htm }}$

17. Op. cit. Perceval Verde. JM.

18. Associació de donnes periodistas. Metgesses, bona salud profesional. № 14 . Barcelona. 2004

19. Perceval Verde, J.M; Tejedor Calvo, S. Tópicos y arquetipos en la red. El otro fantasma virtualmente omnipresente. Barcelona. 2007 